\title{
ESTIMADORES NEW1 E NEW2 NO ESTUDO DA DEPENDÊNCIA ESPACIAL DA PRODUTIVIDADE DA SOJA E ATRIBUTOS FÍSICOS DO SOLO EM UMA ÁREA COMERCIAL ${ }^{1}$
}

\author{
Aracéli Ciotti de Marins²; Miguel Angel Uribe-Opazo³; Jerry Adriani Johann ${ }^{4}$
}

\begin{abstract}
RESUMO
A geoestatística tem como princípio conhecer a estrutura de dependência espacial de variáveis regionalizadas pela função semivariância, cujos estimadores podem ser: Clássico de Matheron, Cressie \& Hawkins e o de Semivariância Relativa Pairwise. Li \& Lake (1994) propuseram dois novos estimadores chamados New1 e New2, os quais, segundo os autores, são mais eficientes que os demais. Neste trabalho, os cinco estimadores citados foram comparados pela aplicação referente aos dados da resistência do solo à penetração, densidade do solo, umidade volumétrica e produtividade da soja, em uma área comercial de $47 \mathrm{ha}$, para determinar a estrutura de dependência espacial, construir mapas temáticos e ser utilizada na Agricultura de Precisão. Apenas os estimadores New1 e New2 detectaram estrutura de dependência espacial entre os pontos amostrais para as variáveis em estudo.
\end{abstract}

Palavras-chave: Geoestatística, variáveis regionalizadas, agricultura de precisão.

\section{ABSTRACT \\ New1 and New2 Estimators on Spatial Dependence Study Regarding Soybean Yield and Soil Physical Attributes in a Commercial Area}

The basic aim of Geostatistics is to understand the spatial dependence structure of regional variables by semivariance function, whose estimators can be the Classical Semivariance of Matheron, the Cressie \& Hawkins and the Pairwise Relative Semivariance one. Thus, Li \& Lake (1994) proposed two new estimators (New1 and New2), which are considered more efficient than the others. In this study, the five estimators were compared by applying them to the data of soil resistance to penetration, bulk density, soil moisture and soybean yield, in a 47 ha commercial farm, to determine the spatial dependence structure, construct thematic maps and to be use in Precision Agriculture. Only New1 and New2 estimators detected spatial dependence structures among the sampling points for the variables analyzed.

Keywords: Geostatistics, regional variables, precision agriculture.

\footnotetext{
Recebido para publicação em 04.05.2006

${ }^{1}$ Parte da dissertação do primeiro autor.

${ }^{2}$ Prof. Msc. da Universidade Tecnológica Federal do Paraná - Campus de Campo Mourão, email: araceli@ utfpr.edu.br.

${ }^{3}$ Prof. Dr. do Centro de Ciências Exatas e Tecnológicas - UNIOESTE - Cascavel, email: mopazo@unioeste.br.

${ }^{4}$ Prof. Msc. do Centro de Ciências Exatas e Tecnológicas - UNIOESTE - Cascavel, email: jerryaj@ pop.com.br.
} 


\section{INTRODUÇÃO}

A agricultura é um dos setores mais crescentes da economia mundial e vem sendo desenvolvida de forma a aprimorar suas técnicas, visando o aumento da produtividade $e$ qualidade do que é cultivado, sendo que, com esta crescente expansão da agricultura, é indispensável encontrar melhores formas para realizar o plantio e o manejo de culturas.

O produtor costuma tratar cada setor de sua lavoura de acordo com suas particularidades, porém, quando se trata de áreas comerciais, isto se torna inviável, devido ao grande número de operações necessárias. Para solucionar esta dificuldade, surgiu a Agricultura de Precisão - AP que propicia meios para que cada setor da lavoura seja tratada de forma diferenciada, garantindo o tratamento necessário e suficiente sem afetar o meio ambiente.

No estudo da produtividade das culturas, é necessário estudar a variabilidade espacial dos atributos do solo, podendo-se aplicar os insumos nos locais necessários, buscando uma maior e homogênea produtividade em toda a área de cultivo.

Um dos métodos utilizados para realizar esta regionalização é a Geoestatística, definida como um conjunto de técnicas e procedimentos estatísticos aplicados a variáveis regionalizadas, que definem a estrutura de dependência espacial de cada variável, em que os dados são referenciados espacialmente. Desta forma, cada ponto amostral é analisado, além de seu valor, de acordo com a posição geográfica em que se encontra, sendo que este fato faz com que amostras próximas tenham valores mais semelhantes e sejam mais correlacionadas do que amostras mais distantes do ponto referencial. Assim, podem-se estimar os valores dos atributos do solo e/ou produtividade das culturas em locais não amostrados pela técnica geoestatística de interpolação por Krigagem. Com os valores estimados e os amostrados, constroem-se mapas temáticos dos atributos do solo que, além de permitirem o entendimento da variabilidade espacial, permitem variar a taxa de aplicação de insumos na área de plantio, com a finalidade de obter aumento em produtividade.

No estudo geoestatístico da estrutura de dependência espacial, comumente utiliza-se o semivariograma, um gráfico da distância versus a função semivariância, que verifica a existência ou não de uma estrutura de dependência espacial nas amostras, por meio de um estimador da função semivariância a diferentes distâncias (Isaaks \& Srivastava, 1989). A função semivariância real é desconhecida, mas pode ser estimada. Entretanto, a maioria dos estimadores utilizados não é robusta a pontos discrepantes ou a dados cuja distribuição não seja normal. Assim, justifica-se 0 estudo de novos estimadores, para estimativa mais adequada da semivariância real.

A função semivariância $\gamma(h)$ é definida como a metade da esperança matemática do quadrado da diferença do valor da função aleatória ou variável regionalizada $Z$ verificada em dois pontos separados, no espaço ou no tempo, por um vetor $h$

$$
\gamma(h)=\frac{1}{2} E[Z(s)-Z(s+h)]^{2}
$$

em que $s \in S \subset R^{2}$.

Os estimadores mais conhecidos da função semivariância, definida na Equação (1), são: o Estimador Clássico de Matheron (Matheron, 1963), o Estimador de Cressie \& Hawkins (Cressie \& Hawkins, 1980) e o Estimador de Semivariância Relativa Pairwise (Srivastava \& Parker, 1989).

O Estimador Clássico de Matheron é expresso como

$\hat{\gamma}_{M}(h)=\frac{1}{2 N(h)} \sum^{N(h)}[Z(s)-Z(s+h)]^{2}$

em que

$N(h)=$ número de pares de dados utilizados a uma distância $h$;

$Z$ = variável regionalizada ou função aleatória; e

$s=\left(x_{i}, y_{j}\right) \in S \subset R^{2} \quad$ são as posições amostrais.

O estimador da função semivariância, proposto por Cressie \& Hawkins (1980), é dado por

$$
\hat{\gamma}_{C}(h)=\frac{\left\{\frac{1}{N(h)} \sum^{N(h)}|Z(s)-Z(s+h)|^{\frac{1}{2}}\right\}^{4}}{0,914+\frac{0,988}{N(h)}}
$$


O estimador de Matheron $\hat{\gamma}_{M}$ dado na Equação (2) é o mais estável desde que os dados apresentem distribuição normal de probabilidade, entretanto, na presença de uma distribuição de caudas pesadas, o estimador de Cressie \& Hawkins $\hat{\gamma}_{C}$ é mais apropriado (Cressie, 1993).

O estimador de semivariância relativa Pairwise (Srivastava \& Parker, 1989; Isaaks \& Srivastava, 1989) é dado por

$$
\hat{\gamma}_{P}(h)=\frac{2}{N(h)} \sum^{N(h)}\left[\frac{Z(s)-Z(s+h)}{Z(s)+Z(s+h)}\right]^{2}
$$

O estimador de semivariância relativa Pairwise $\hat{\gamma}_{P}$, apresentado na Equação (4), caracteriza-se por diminuir o efeito de dados discrepantes, porém apresenta a desvantagem da soma dos valores da variável $Z$ nos pontos de coordenadas $s$ e $s$ $+h$ não poder ser nula; além disso, quando a média se aproxima de zero, podem ocorrer semivariogramas com "saltos" nas semivariâncias (Isaaks \& Srivastava, 1989).

Dois novos estimadores de semivariância, New1 e New2, foram propostos por Li \& Lake (1994), visando reduzir a imprecisão do estimador, quando a distância entre as amostras aumenta. Os estimadores da função semivariância New1 e New2 são dados respectivamente pelas equações (5) e (6)

$$
\begin{aligned}
& \hat{\gamma}_{N 1}(h)=\frac{1}{n} \sum_{i=1}^{n}\left\{\frac{1}{2 m} \sum_{j \in D_{i, h}}\left[Z\left(s_{i}\right)-Z\left(s_{j}\right)\right]^{2}\right\} \\
& \hat{\gamma}_{N 2}(h)=\hat{\gamma}_{N 1}(h)+\frac{h}{d} \hat{\gamma}_{N 1}^{\prime}(h)
\end{aligned}
$$

em que

$\mathrm{n}$ = número de pontos amostrais na área toda;

$D_{\mathrm{i}, \mathrm{h}}=$ conjunto indexado dos valores da variável regionalizada $Z$, em pontos de uma janela móvel bi-dimensional de área $\Delta_{i, h}$ com tamanho $h$ centrada no bloco i, excluindo o ponto $\mathrm{s}_{\mathrm{i}}$;

$\mathrm{m}=$ número de dados em $D_{\mathrm{i}, \mathrm{h}}$;

$\hat{\gamma}_{N 1}^{\prime}(h)=$ valor da derivada da função $\hat{\gamma}_{N 1}$ na distância $h$; sendo $\hat{\gamma}_{N 1}$ o modelo ajustado a $\hat{\gamma}_{N 1}(h) ; \mathrm{e}$ $d=$ momento da semivariância $(d=\bar{X}$ ou $\left.d=S^{2}+\bar{X}^{2}\right)$.

No ajuste de um semivariograma teórico a um semivariograma experimental, tem-se o modelo e seus três parâmetros, que definem a estrutura de dependência espacial: efeito pepita $\left(\mathrm{C}_{0}\right)$, que corresponde ao valor do semivariograma, quando a distância $h$ é igual a zero; patamar $\mathrm{C}=\left(\mathrm{C}_{0}+\mathrm{C}_{1}\right)$, que é o maior valor do semivariograma, em que este se estabiliza e corresponde ao alcance $(a)$, que é a distância, dentro da qual as amostras apresentam dependência espacial. É a partir do alcance (a) que se considera a inexistência de dependência espacial entre as amostras, sendo possível o estudo de dados por meio da estatística clássica, que tem como base a independência dos dados.

Quando a variável regionalizada apresenta estrutura de dependência espacial com patamar, o grau de dependência espacial pode ser determinado pelo coeficiente de efeito pepita relativo, apresentado na equação

$\varepsilon=\frac{C_{0}}{C_{0}+C_{1}}$

Segundo Souza et al. (1999), a dependência espacial é considerada forte, quando o coeficiente de efeito pepita é menor que 0,25. Quando o coeficiente de efeito pepita está entre 0,25 e 0,75 , a dependência é considerada moderada e, acima de 0,75 , é considerada fraca.

O objetivo deste trabalho foi verificar a existência de uma estrutura de dependência espacial, utilizando-se os estimadores de semivariância de Matheron (Matheron, 1963), Cressie \& Hawkins (Cressie \& Hawkins, 1980), Pairwise (Srivastava \& Parker, 1989; Issaaks \& Srivastava,1989), New1 e New2 (Li \& Lake, 1994) nos atributos físicos do solo: densidade, umidade volumétrica e resistência do solo à penetração nas profundidades de $0-10,10-20$ e $20-30 \mathrm{~cm}$ e na produtividade da soja em uma área comercial de 47 ha, com uma grade amostral regular de $100 \times 100 \mathrm{~m}$. 


\section{MATERIAL E MÉTODOS}

O experimento foi instalado na área comercial cuja latitude é 24050'19,81"S, longitude 5336'33,30"O e altitude 659,5 m, município de Cascavel, Estado do Paraná. O solo da área é classificado como Latossolo Vermelho Escuro distroférrico, derivado de rochas de origem basáltica, apresentando relevo suavemente ondulado. A precipitação pluviométrica anual média é de $1.940 \mathrm{~mm}$, com maiores concentrações durante os meses de janeiro, maio e outubro, com períodos mais secos em junho e agosto. O clima é temperado mesotérmico e superúmido, temperatura anual média de $21^{\circ} \mathrm{C}$, altitude média de $800 \mathrm{~m}$. O sistema de manejo é o plantio direto. Os pontos amostrais foram georreferenciados com o auxílio de um GPS, sendo 48 pontos dispostos em uma grade regular de $100 \times 100 \mathrm{~m}$.

Foram estudadas as seguintes variáveis regionalizadas: densidade do solo - ds

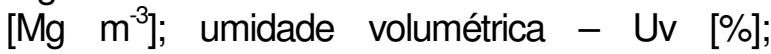
resistência do solo à penetração - RSP [MPa]; e produtividade da soja [Mg ha-1].

As amostras para determinação da densidade e umidade volumétrica do solo, foram retiradas em três profundidades: $0-10 \mathrm{~cm}, 10-20 \mathrm{~cm}$ e 20 $30 \mathrm{~cm}$, em cada ponto amostral. Foram feitas duas repetições a cada profundidade, a fim de obter amostras representativas, considerando-se a grande dispersão destes atributos físicos e para prevenir perda de amostras durante a realização dos procedimentos laboratoriais.

Para determinação da densidade do solo - ds [Mg m$\left.{ }^{-3}\right]$, utilizou-se o método do anel volumétrico e, para determinar a umidade volumétrica do solo - Uv [\%], utilizou-se o método da secagem em estufa para determinar a umidade gravimétrica, ambos descritos por EMBRAPA (1997).

A RSP [MPa] foi determinada na profundidade de $0-30 \mathrm{~cm}$ e foram realizadas dez repetições em cada um dos pontos amostrais, sendo que foi coletada por meio de um penetrógrafo de haste, com ângulo de cone de $30^{\circ}$, diâmetro da base de cone de $12,83 \mathrm{~mm}$, diâmetro da haste de $9,53 \mathrm{~mm}$ e comprimento de $600 \mathrm{~mm}$ descrito em Tieppo (2004).

A partir do conjunto de dados obtidos calculo-se o índice de cone para as camadas de $0-10 \mathrm{~cm}, 10-20 \mathrm{~cm}$ e $20-30 \mathrm{~cm}$.
A produtividade da soja $\left[\mathrm{Mg} \mathrm{ha}{ }^{-1}\right]$ foi verificada pela colheita em duas linhas, em um comprimento de $1,5 \mathrm{~m}$ em cada um dos pontos amostrais. A soja foi trilhada e pesada. Utilizou-se fator de correção de umidade $f c$ de $13 \%$. O valor de $f c(\%)$ é obtido pela equação

$f c=\frac{U A-U D}{U D} \times 100$

em que

$U A=$ é a umidade atual [\%]; e

$U D=$ é a umidade desejada [\%].

Para o estudo de estacionaridade e continuidade espacial, foram construídos gráficos de tendência (Post-plot) e gráficos de dispersão de médias móveis versus desvios-padrão, que foram utilizados para verificar se os dados apresentavam tendência direcional. Aos dados que apresentaram tendência direcional, trabalhou-se com os resíduos obtidos pela aplicação da técnica de regressão em função das coordenadas $(X, Y)$.

Implementaram-se, computacionalmente, as funções semivariância de Matheron, Cressie \& Hawkins, Pairwise, New1 e New2, sendo que os valores obtidos foram utilizados para elaboração de mapas temáticos pelo método de interpolação por Krigagem ordinária, para as variáveis regionalizadas que apresentaram estrutura de dependência espacial.

\section{RESULTADOS E DISCUSSÃO}

No Quadro 1, observa-se que os valores da densidade média (ds) nas profundidades 0-10 e $20-30 \mathrm{~cm}$ foram praticamente iguais, sendo que, nestas profundidades, a densidade média é maior que na profundidade de $10-20 \mathrm{~cm}$. A densidade maior na profundidade de $20-30 \mathrm{~cm}$ justifica que a resistência do solo a penetração (RSP) tenha sido maior nesta profundidade, pois, segundo Araújo et al. (2004), a densidade influencia os resultados de RSP. Como os coeficientes de variação da densidade nas três profundidades são menores que $10 \%$, considera-se que os dados de densidade apresentam homogeneidade (Gomes, 1987). 
Quadro 1. Análise Exploratória de dados das variáveis regionalizadas em estudo

\begin{tabular}{|c|c|c|c|c|c|c|c|c|}
\hline $\begin{array}{l}\text { Variáveis } \\
\text { regionalizadas }\end{array}$ & Média & $\begin{array}{l}\text { Desvio- } \\
\text { padrão }\end{array}$ & $\begin{array}{c}\text { Coeficiente } \\
\text { de } \\
\text { variação } \\
(\%)\end{array}$ & $\begin{array}{c}1^{10} \\
\text { Quartil }\end{array}$ & Mediana & $\begin{array}{c}3^{\circ} \\
\text { Quartil }\end{array}$ & $\begin{array}{l}\text { Valor } \\
\text { mínimo }\end{array}$ & $\begin{array}{l}\text { Valor } \\
\text { máximo }\end{array}$ \\
\hline $\mathrm{ds} 1\left[\mathrm{Mg} \mathrm{m}^{-3}\right]$ & 1,19 & 0,05 & 4,26 & 1,170 & 1,210 & 1,228 & 1,060 & 1,290 \\
\hline ds2 $\left[\mathrm{Mg} \mathrm{m}^{-3}\right]$ & 1,16 & 0,09 & 7,60 & 1,100 & 1,150 & 1,200 & 1,040 & 1,550 \\
\hline $\mathrm{ds} 3\left[\mathrm{Mg} \mathrm{m}^{-3}\right]$ & 1,20 & 0,06 & 5,15 & 1,160 & 1,205 & 1,248 & 1,100 & 1,350 \\
\hline Uv1 [\%] & 0,37 & 0,04 & 10,53 & 0,343 & 0,360 & 0,390 & 0,260 & 0,500 \\
\hline Uv2 [\%] & 0,36 & 0,03 & 8,44 & 0,340 & 0,360 & 0,380 & 0,300 & 0,490 \\
\hline Uv3 [\%] & 0,37 & 0,03 & 6,99 & 0,360 & 0,370 & 0,388 & 0,290 & 0,420 \\
\hline $\mathrm{RSP} 1$ [MPa] & 2,64 & 0,63 & 23,70 & 2,154 & 2,630 & 3,024 & 1,538 & 4,391 \\
\hline RSP2 [MPa] & 3,30 & 0,86 & 25,90 & 2,731 & 3,194 & 3,880 & 0,921 & 4,910 \\
\hline RSP3 [MPa] & 3,30 & 0,68 & 20,60 & 2,794 & 3,113 & 3,763 & 2,071 & 4,929 \\
\hline Prod $\left[\mathrm{Mg} \mathrm{ha}^{-1}\right]$ & 1,87 & 0,46 & 24,57 & 1,650 & 1,790 & 2,110 & 0,960 & 3,970 \\
\hline
\end{tabular}

Prod: produtividade da soja; ds1: densidade do solo de 0-10 cm; ds2: densidade do solo de 10-20 cm; ds3: densidade do solo de 20-30 cm; Uv1: umidade volumétrica de 0-10 cm; Uv2: umidade volumétrica de 10-20 cm; Uv3: umidade volumétrica de 20-30 cm; RSP1: RSP de 0-10 cm; RSP2: RSP de 10-20 cm; RSP3: RSP de 20-30 cm.

No Quadro 1, observa-se que na umidade volumétrica (Uv), $75 \%$ dos dados (no 3ำ Quartil) têm menores valores nas profundidades de $10-20 \mathrm{~cm}$ e $20-30 \mathrm{~cm}$. Segundo Secco (2003), isto ocorre, pois os dados de RSP apresentam relação inversa com os dados de umidade do solo. Devido ao alto coeficiente de variação da RSP nas três nas profundidades, os dados foram considerados heterogêneos em relação a sua média (Gomes, 1987). A produtividade média da soja (Prod) de $1,87 \mathrm{Mg} \mathrm{ha}^{-1}$ foi considerada baixa, de acordo com EMBRAPA (2004), que considera baixa uma produtividade menor que $2,5 \mathrm{Mg} \mathrm{ha}^{-1}$. A produtividade foi baixa, também em comparação com a produtividade do município de Cascavel e do Estado do Paraná, pois, segundo PARANÁ (2005), a produtividade média da soja no município de Cascavel, no ano agrícola $2004 / 2005$, foi de $2,75 \mathrm{Mg} \mathrm{ha}^{-1}$ e no Estado do Paraná de 2,56 $\mathrm{Mg} \mathrm{ha-1}$. Devido ao alto coeficiente de variação da produtividade $(24,57 \%)$, os dados foram considerados heterogêneos em relação à sua média (Gomes, 1987).

No Quadro 2, são apresentados resultados dos estudos de variabilidade espacial das variáveis regionalizadas. Os estimadores da função semivariância New1 e New2 detectaram estrutura de dependência espacial entre as amostras com uma grade amostral de $100 \times 100 \mathrm{~m}$ (uma amostra por hectare) e distâncias de até $50 \%$ (cutoff) da distância máxima entre pontos, sendo que os estimadores de Matheron, Cressie \& Hawkins e Pairwise não detectaram essas dependências espaciais.

Segundo o estimador New1, a função semivariância experimental dos dados densidade do solo na profundidade de $0-10$ $\mathrm{cm}$ (ds1) apresentaram estrutura de dependência espacial moderada $(25 \%<\varepsilon \leq$ $75 \%)$ por meio do ajuste de um modelo gaussiano, utilizando um ponto de corte (cutoff) de $25 \%$ da distância máxima. Assim, em um raio de $300 \mathrm{~m}$, os dados são correlacionados espacialmente. A Figura 1(a) apresenta o semivariograma experimental, obtido pelo estimador New1, enquanto a Figura 1(b) apresenta o mapa temático da densidade do solo à profundidade de $0-10 \mathrm{~cm}$, com os parâmetros $\mathrm{C}_{0}, \mathrm{C}_{1}$ e a apresentados no Quadro 2.

Observa-se na Figura 1(b), que a maior parte da área tem densidade entre 1,17 e $1,19 \mathrm{Mg} \mathrm{m}^{-3}$, sendo pequena a área com alta densidade, de $1,24 \mathrm{Mg} \mathrm{m}^{-3}$. 
Quadro 2. Resultados da análise geoestatística das variáveis regionalizadas

\begin{tabular}{ccccccc}
\hline Variável & Estimador & Modelo & $\mathrm{C}_{0}$ & $\mathrm{C}_{1}$ & $a$ & $\varepsilon(\%)$ \\
\hline $\mathrm{ds} 1\left[\mathrm{Mg} \mathrm{m}^{-3}\right]$ & New1 & Gaussiano & 0,0027 & 0,0028 & 300 & 49,0 \\
ds2 $\left[\mathrm{Mg} \mathrm{m}^{-3}\right]$ & New2 & Esférico & 0,0010 & 0,2070 & 310 & 0,5 \\
ds3 $\left[\mathrm{Mg} \mathrm{m}^{-3}\right]$ & New2 & Exponencial & 0,0005 & 0,3900 & 435 & 0,128 \\
Uv1 [\%] & New1 & Gaussiano & 0,0012 & 0,0002 & 300 & 85,71 \\
Uv2 [\%] & New1 & Esférico & 0,0006 & $3 \times 10^{-5}$ & 165 & 96,0 \\
Uv3 [\%] & New1 & Esférico & $1 \times 10^{-5}$ & 0,0009 & 150 & 1,1 \\
RSP1 [MPa] & New2 & Esférico & $1 \times 10^{-5}$ & 150 & 550 & 0,0000007 \\
RSP2 [MPa] & New2 & Esférico & 0,5000 & 0,0800 & 200 & 86,0 \\
RSP3 [MPa] & New2 & Esférico & $1 \times 10^{-5}$ & 0,8500 & 300 & 0,0012 \\
Prod $[\mathrm{Mg} \mathrm{ha-1}]$ & New2 & Esférico & $1 \times 10^{-4}$ & 0,3300 & 400 & 0,03
\end{tabular}

Prod: produtividade da soja; ds1: densidade do solo de 0-10 cm; ds2: densidade do solo de 10-20 cm; ds3: densidade do solo de 20-30 cm; Uv1: umidade volumétrica de 0-10 cm; Uv2: umidade volumétrica de 10-20 cm; Uv3: umidade volumétrica de 20-30 cm; RSP1: RSP de 0-10 cm; RSP2: RSP de 10-20 cm; RSP3: RSP de 20-30 cm; $\mathrm{C}_{0}$ : efeito pepita; $\mathrm{C}_{1}$ : contribuição; $a$ : alcance; $\varepsilon$ : coeficiente de efeito pepita

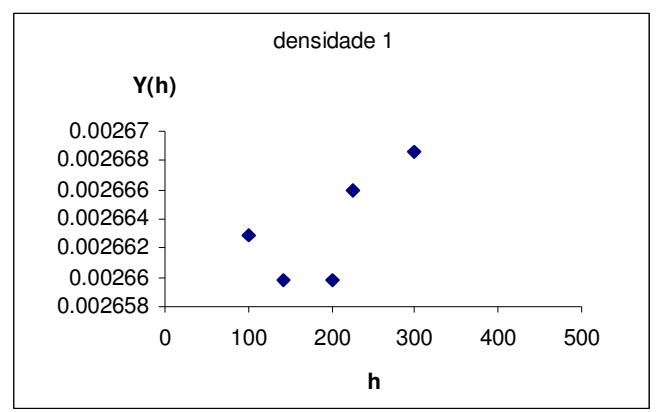

(a)

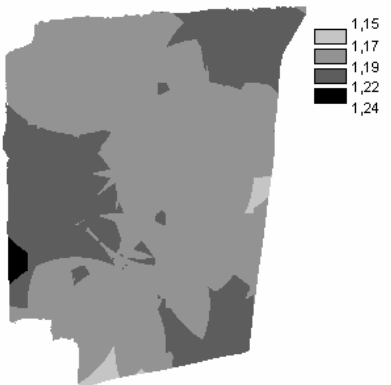

(b)

Figura 1. (a) Semivariograma experimental da densidade do solo de 0-10 cm, segundo o estimador New1; (b) mapa temático da densidade do solo na profundidade de 0 $10 \mathrm{~cm}$

As Figuras 2(a) e (b) foram construída utilizando-se os parâmetros apresentados no Quadro 2, segundo o qual, a densidade do solo na profundidade de $10-20 \mathrm{~cm}$ (ds2) apresenta dependência espacial forte $(\varepsilon<$ $25 \%$ ) através do ajuste de um modelo esférico com um raio de dependência espacial entre amostras de $310 \mathrm{~m}$, por meio do estimador New2. A Figura 2(a) apresenta o semivariograma experimental e na Figura 2(b) apresenta-se o mapa temático da densidade do solo na profundidade de 10-20 $\mathrm{cm}$. Observa-se na Figura 2(b) que mais de $50 \%$ da área apresenta densidade menor que $1,16 \mathrm{Mg} \mathrm{m}^{-3}$ na profundidade de $10-20$ $\mathrm{cm}$.

No Quadro 2 verifica-se segundo 0 estimador New2 que a densidade do solo na profundidade de $20-30 \mathrm{~cm}$ (ds3) apresenta dependência espacial forte $(\varepsilon<25 \%)$ através do ajuste de um modelo Exponencial, com raio de dependência entre amostras de $435 \mathrm{~m}$. Assim, foram construídos o semivariograma experimental na Figura 3(a) e o mapa temático desta variável na Figura 3(b). Verifica-se, pela Figura 3(b), que a maior parte da área em estudo tem densidade do solo menor que 1,17

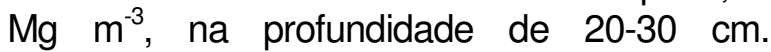




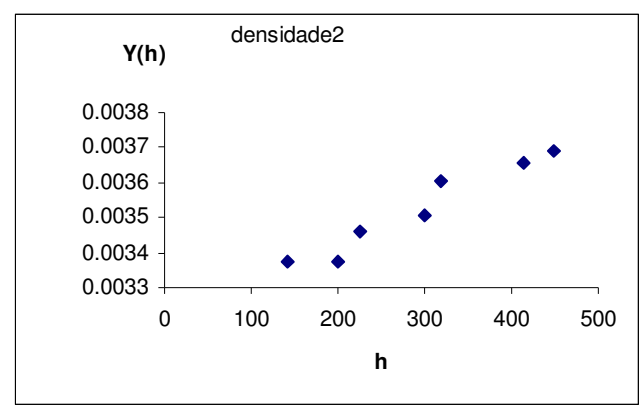

(a)

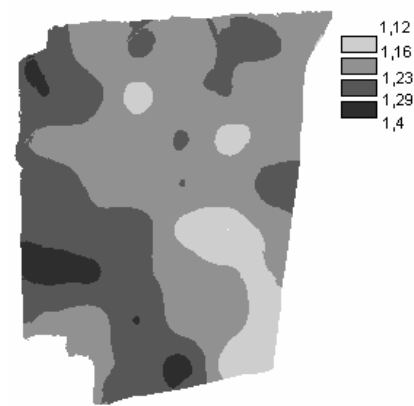

(b)

Figura 2. (a) Semivariograma experimental da densidade do solo na profundidade de 10-20 $\mathrm{cm}$ segundo os estimador New2, (b) Mapa temático da densidade do solo $10-20 \mathrm{~cm}$

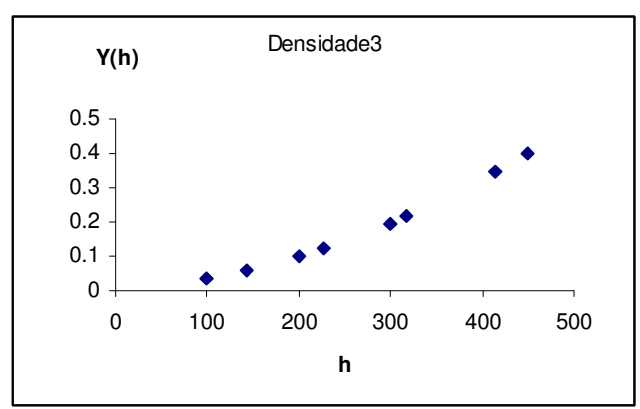

(a)

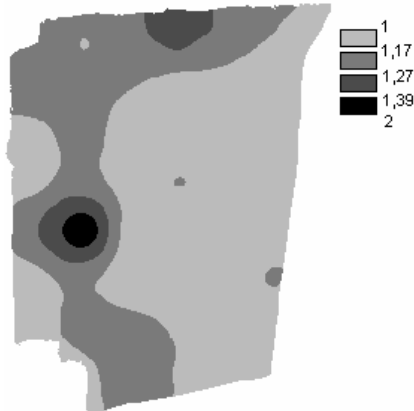

(b)

Figura 3. (a) Semivariogramas experimental da densidade do solo na profundidade de $20-30 \mathrm{~cm}$ segundo o estimador New2, (b) mapa temático da densidade do solo de $20-30 \mathrm{~cm}$

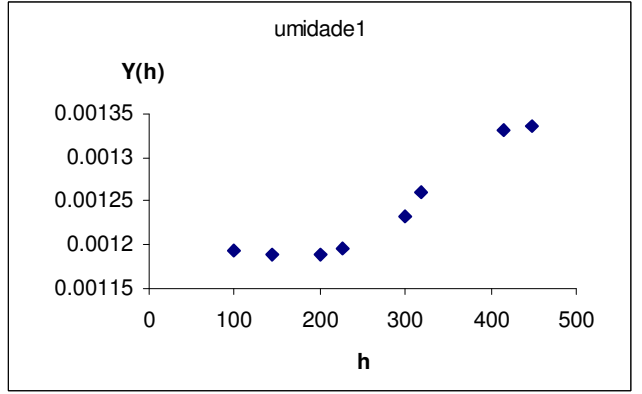

(a)

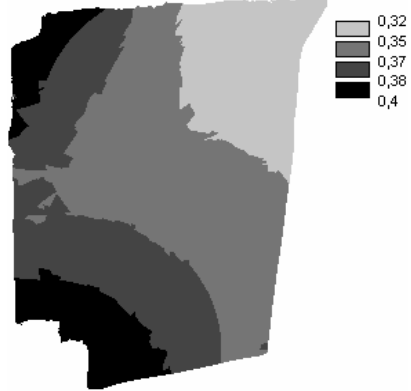

(b)

Figura 4. (a) Semivariogramas experimental da umidade volumétrica na profundidade de $0-10 \mathrm{~cm}$ segundo o estimador New1, (b) Mapa temático da umidade volumétrica de 0-10 cm

A estrutura de dependência espacial da umidade volumétrica na profundidade de $0-10 \mathrm{~cm}$ (Uv1) é fraca $(\varepsilon>75 \%)$ e atende ao modelo gaussiano pelo estimador New1 (Quadro 2). A Figura 4(a) apresenta 0 semivariograma experimental construído pelo estimador New1 e a Figura 4(b) apresenta o mapa temático da umidade volumétrica na profundidade de $0-10 \mathrm{~cm}$.

Comparando as Figuras 1(b) e 4(b) na profundidade de $0-10 \mathrm{~cm}$, verifica-se que os locais onde há alta umidade volumétrica a densidade do solo é maior, já nos locais de baixa umidade volumétrica, a densidade do solo é alta.

A umidade volumétrica de $10-20 \mathrm{~cm}$ apresenta segundo o estimador New1, estrutura de dependência espacial fraca $(\varepsilon$ $>75 \%$ ) obtida pelo modelo esférico. A Figura 5(a) apresenta o semivariograma construído a partir dos parâmetros apresentados no Quadro 2 e a Figura 5(b) apresenta o mapa temático da umidade volumétrica de $10-20 \mathrm{~cm}$. 
A estrutura de dependência espacial da umidade volumétrica de $20-30 \mathrm{~cm}$ (Uv3) apresentada no Quadro (2) é forte $(\varepsilon<25 \%)$ e atende ao modelo esférico pelo estimador New1. Devido ao comportamento da semivariância experimental foi utilizado um cutoff de $16 \%$, e obteve-se um alcance de $150 \mathrm{~m}$. A Figura 6(a) apresenta o semivariograma experimental obtido pelo estimador New1 e a Figura 6(b) apresenta o mapa temático da umidade volumétrica de 20-30 cm.

A RSP na camada de $0-10 \mathrm{~cm}$ apresenta estrutura de dependência espacial forte $(\varepsilon<$ $25 \%$ ) com um modelo teórico ajustado esférico segundo o estimador New2. A Figura $7($ a) apresenta 0 semivariograma experimental construído pelo estimador New2 e a Figura 7(b) apresenta seu respectivo mapa temático da RSP na camada de 0-10 cm, construídos com os parâmetros apresentados no Quadro 2.

No Mapa temático apresentado na Figura 7(b) observa-se uma baixa RSP na camada de

0-10 cm, verificando no canto superior esquerdo, istó deve-se a uma baixa densidade do solo no mesmo local, nesta profundidade, como é apresentado na Figura 1(b).

No Quadro (2) verificou-se que a RSP na camada de $10-20 \mathrm{~cm}$ apresenta uma estrutura de dependência espacial fraca $(\varepsilon>$ $75 \%$ ) obtida pelo modelo esférico com alcance de $200 \mathrm{~m}$. A Figura 8(a) apresenta o semivariograma experimental obtido pelo estimador New2 e a Figura 8(b) apresenta o mapa temático da RSP na profundidade de 10-20 cm, construídos conforme parâmetros apresentados no Quadro 2.

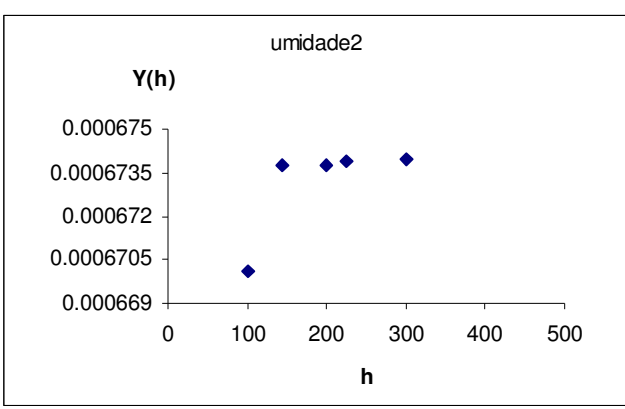

(a)

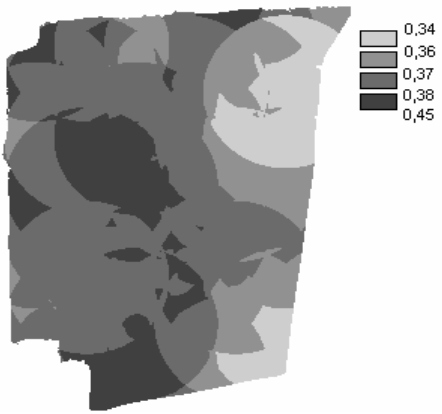

(b)

Figura 5. (a) Semivariogramas experimental da umidade volumétrica na profundidade de 10-20 cm segundo o estimador New1, (b) Mapa temático da umidade volumétrica de $10-20 \mathrm{~cm}$

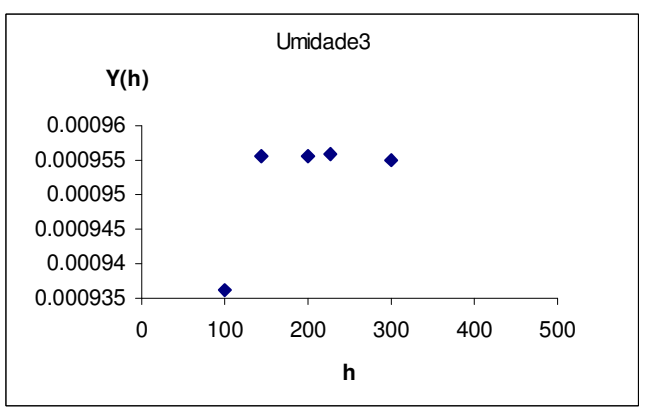

(a)

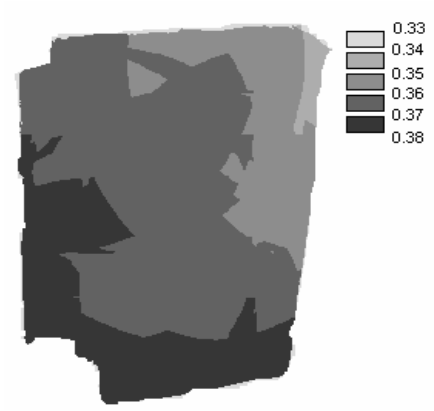

(b)

Figura 6. (a) Semivariograma experimental da umidade volumétrica na profundidade de 20$30 \mathrm{~cm}$ segundo o estimador New1, (b) mapa temático da umidade volumétrica de $20-30 \mathrm{~cm}$ 


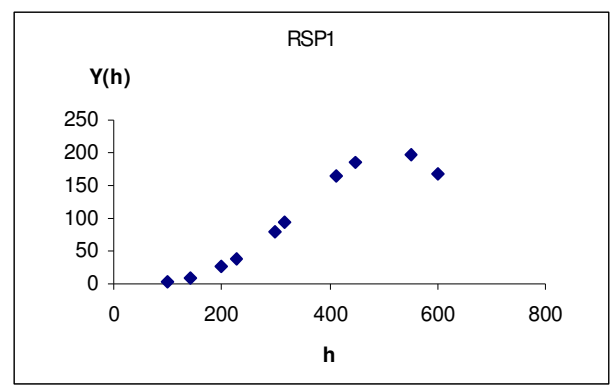

(a)

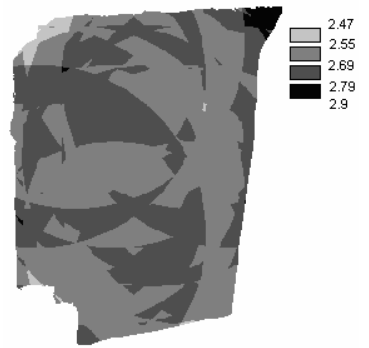

(b)

Figura 7. (a) Semivariograma experimental da RSP na camada de 0-10 cm obtido pelo estimador New2, (b) mapa temático da RSP na camada de 0-10 cm

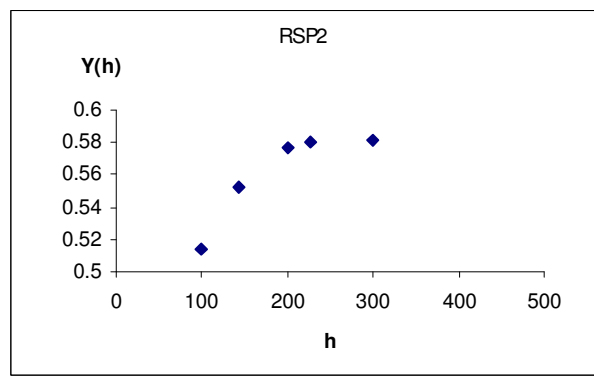

(a)

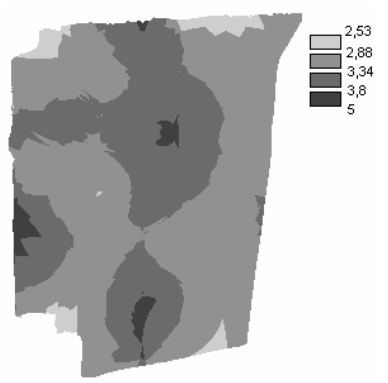

(b)

Figura 8. (a) Semivariograma experimental da RSP na camada de $10-20 \mathrm{~cm}$ segundo o estimador New2, (b) mapa temático da RSP na camada de $10-20 \mathrm{~cm}$

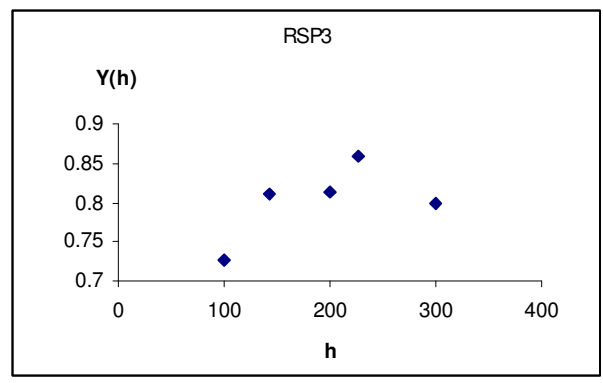

(a)

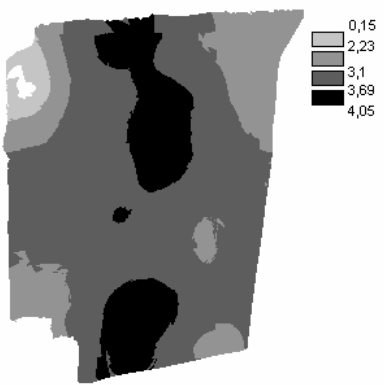

(b)

Figura 9. (a) Semivariograma experimental da RSP na camada de $20-30 \mathrm{~cm}$ segundo o estimador New2, (b) mapa temático da RSP na camada de $20-30 \mathrm{~cm}$

Comparando os mapas temáticos das Figuras 6(b) e 9(b), verifica-se que nos locais onde há uma alta RSP, a umidade volumétrica é baixa, na camada de 10-20 $\mathrm{cm}$.

Segundo o Quadro (2) a RSP na profundidade $20-30 \mathrm{~cm}$ segundo o estimador New2, os dados apresentam estrutura de dependência espacial forte $(\varepsilon<25 \%)$, segundo o modelo esférico com raio de 300 m. As Figuras 9(a) e 9(b) foram construídas utilizando-se os parâmetros apresentados no Quadro 2.
Observa-se, na Figura 9(b) que há uma região (canto superior esquerdo) onde a RSP na profundidade de $20-30 \mathrm{~cm}$ é a mais baixa de toda a área, e isso ocorre devido à alta RSP nas camadas de 0-10 e 20-30 cm, que repercutem num aumento da RSP na camada inferior.

A Figura 10(a) apresenta o semivariograma experimental e a Figura 10(b) apresenta o mapa temático da produtividade da soja, com parâmetros obtidos no Quadro (2), segundo o qual os dados de produtividade da soja, apresentam estrutura de dependência espacial forte $(\varepsilon<25 \%)$, obtida pelo modelo esférico com alcance de $400 \mathrm{~m}$. 


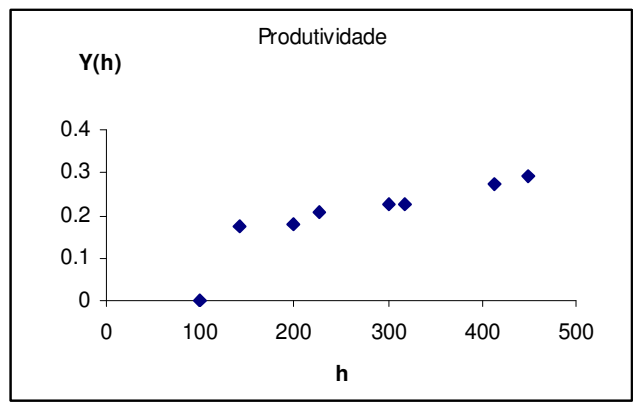

(a)

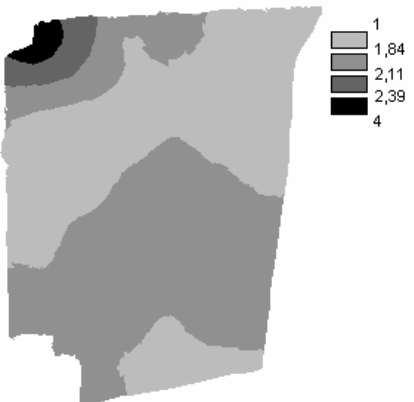

(b)

Figura 10. (a) Semivariograma experimental da variável produtividade da soja segundo o estimador New2, (b) mapa temático da produtividade da soja

Observa-se na Figura 10(b) que o canto superior esquerdo é o local onde se verificou produtividade máxima de $3,97 \mathrm{Mg} \mathrm{ha}^{-1}$. Neste local há baixa densidade do solo segundo as Figuras 1(b) e 3(b) e baixa RSP segundo as Figuras 7(b), 8(b) e 9(b).

\section{CONCLUSÕES}

- Os estimadores New1 e New2 detectaram estrutura de dependência espacial entre as amostras das variáveis regionalizadas em estudo, onde os estimadores Matheron, Cressie \& Hawkins e Pairwise não detectaram.

- Com uma grade de 100 x 100 m pode-se observar que apenas os estimadores New1 e New2 detectam estrutura de dependência espacial, conseqüentemente para trabalhar com esta distância mínima entre os pontos, deve-se utilizar sub-amostras aleatórias retiradas de pontos definidos da grade, se o objetivo for utilizar os demais estimadores.

\section{AGRADECIMENTOS}

A CAPES, CNPq e Fundação Araucária pelo apoio financeiro, à Fazenda Wypych eà COODETEC pelo apoio técnico e aos pesquisadores do Laboratório de Estatística Aplicada -LEA.

\section{REFERÊNCIAS BIBLIOGRÁFICAS}

ARAÚUO, M.A.; TORMENA, C.A.; SILVA, A.P. Propriedades físicas de um latossolo vermelho distrófico cultivado sob mata nativa. Revista Brasileira de Ciências do Solo, Viçosa, v. 28, p.337-345, 2004.
CRESSIE, N. Statistics for spatial data. New York: Jonh Wiley, 1993. 900 p.

CRESSIE, N.; HAWKINS, D. M. Robust estimation of the variogram, I. v.12, n. 2. Mathematical Geology. New York, p. 115125, 1980.

EMPRESA BRASILEIRA DE PESQUISA AGROPECUÁRIA - EMBRAPA. Serviço Nacional de Levantamento e Conservação de Solos. Manual de métodos de análise do solo. Rio de Janeiro: EMBRAPA. 1997, 212 p.

EMPRESA BRASILEIRA DE PESQUISA AGROPECUÁRIA - EMBRAPA. Centro Nacional de Pesquisa de Solos. Agricultura de precisão para a recomendação de adubação da soja sob plantio direto. Disponível em: http://www.cnps.embrapa.br/search/pesqs/pr oj 04/proj 04.html. Acesso em: 29/07/2004.

GOMES, F.P. Curso de Estatística Experimental. $12^{\underline{a}}$ ed. Piracicaba, SP: Nobel, 1987. 477p.

ISAAKS, E. H.; SRIVASTAVA, R. M. An introduction to applied geoestatísticas. New York: Oxford University Press, 1989. $560 \mathrm{p}$.

LI, D.; LAKE, L. W. A moving window semivariance estimator. Water Resources Research, Austin, v. 30, n. 5, p. 1479-1489, 1994. 
MATHERON, G. Principles Principe's of Geoestatistics. Economic Lacaster, v. 58, p.1246-1266, 1963.

PARANÁ. Secretaria de Estado da Agricultura e do Abastecimento. SEAB. Disponível: em: www.pr.gov.br/seab/deral/'SOJANORMAL'!A 7, Acesso em: 21/12/2005.

SECCO, D. Estados de compactação de dois latossolos sob plantio direto e suas implicações no comportamento mecânico e na produtividade de culturas. Santa Maria, RS, 108f, 2003. Tese (Doutorado em Agronomia) - Universidade Federal de Santa Maria.

SOUZA, E.G.; JOHANN, J.A.; ROCHA, J.U.; RIBEIRO, S.R.A.; SILVA, M.S.; URIBE-
OPAZO, M.A.; MOLIN, J.P.; OLIVEIRA, E.F.; NÓBREGA, L.H.P. Análise da variabilidade espacial dos atributos de um solo em uma área experimental. Parte I: Análise do $\mathrm{P}, \mathrm{K}$, MO e pH. In: Revista Brasileira de Engenharia Agrícola, p. 80-92, 1999.

SRIVASTAVA, R. M.; PARKER, H. M. Robust measures of spatial continuity. In: Geoestatistics, on the $3^{\text {rd }}$. Geostatistical Congress, Holland: Armstrong, p.295-308, 1989.

TIEPPO, R.C. Desenvolvimento de um penetrômetro eletrônico manual. Cascavel, PR, 2004, 133f. Dissertação (Mestrado em Engenharia Agrícola) - Centro de Ciências Exatas, Universidade Estadual do Oeste do Paraná. 